\title{
Targeted exon skipping with AAV- mediated split adenine base editors
}

\author{
Jackson Winter ${ }^{1}$, Alan Luu ${ }^{2,3}$, Michael Gapinske ${ }^{1}$, Sony Manandhar ${ }^{1}$, Shraddha Shirguppe ${ }^{1}$, Wendy S. Woods ${ }^{1}$, \\ Jun S. Song ${ }^{2,3}$ and Pablo Perez-Pinera ${ }^{1,3,4,5}$
}

\begin{abstract}
Techniques for exclusion of exons from mature transcripts have been applied as gene therapies for treating many different diseases. Since exon skipping has been traditionally accomplished using technologies that have a transient effect, it is particularly important to develop new techniques that enable permanent exon skipping. We have recently shown that this can be accomplished using cytidine base editors for permanently disabling the splice acceptor of target exons. We now demonstrate the application of CRISPR-Cas9 adenine deaminase base editors to disrupt the conserved adenine within splice acceptor sites for programmable exon skipping. We also demonstrate that by altering the amino acid sequence of the linker between the adenosine deaminase domain and the Cas9-nickase or by coupling the adenine base editor with a uracil glycosylase inhibitor, the DNA editing efficiency and exon-skipping rates improve significantly. Finally, we developed a split base editor architecture compatible with adeno-associated viral packaging. Collectively, these results represent significant progress toward permanent in vivo exon skipping through base editing and, ultimately, a new modality of gene therapy for the treatment of genetic diseases.
\end{abstract}

\section{Introduction}

Exon splicing is a natural process that occurs during mRNA maturation and results in exclusion of intronic sequences and the assembly of consecutive or nonconsecutive exons from pre-mRNA ${ }^{1}$. The capability to program transcript splicing is highly desirable for synthetic biology and therapeutic applications, specifically for the treatment of monogenic diseases. Since autosomal diseases are often caused by mutations within exons that lead to loss of protein function, removal of the affected exon may provide a therapeutic benefit by enabling translation of truncated protein isoforms free of mutations that are capable of partially fulfilling the physiological role of the full-length protein. Programmable exon skipping has been demonstrated to be an effective

Correspondence: Pablo Perez-Pinera (pablo@illinois.edu)

'Department of Bioengineering, University of Illinois at Urbana-Champaign, Urbana, IL 61801, USA

${ }^{2}$ Department of Physics, University of Illinois at Urbana-Champaign, Urbana, IL 61801, USA

Full list of author information is available at the end of the article. treatment option for diseases such as muscular dystrophies $^{2}$, epidermolysis bullosa ${ }^{3}$, and spinal muscular atrophy ${ }^{4}$.

Conventional targeted exon skipping has been accomplished by directing antisense oligonucleotides (AONs) to splicing regulatory elements in order to block the native splicing machinery and prevent incorporation of the targeted exon into the mature transcript ${ }^{5}$. AONs are typically delivered by local injection and, given their transient nature, necessitate repeated administration to achieve a lasting therapeutic benefit. More recently, the CRISPR-Cas9 genome editing system has been shown to induce permanent exon skipping ${ }^{6}$, which has been harnessed for therapeutic correction of genetic diseases ${ }^{7}$. However, these gene editing approaches rely on introduction of double strand breaks (DSBs) and, while the targeted exons were effectively skipped, repair of DSBs can result in unpredictable phenotypic outcomes ${ }^{8}$, including a DNA damage response involving activation of TP53 that can compromise survival of the edited cells and

\section{(c) The Author(s) 2019}

(c) Open Access This article is licensed under a Creative Commons Attribution 4.0 International License, which permits use, sharing, adaptation, distribution and reproduction cc) in any medium or format, as long as you give appropriate credit to the original author(s) and the source, provide a link to the Creative Commons license, and indicate if changes were made. The images or other third party material in this article are included in the article's Creative Commons license, unless indicated otherwise in a credit line to the material. If material is not included in the article's Creative Commons license and your intended use is not permitted by statutory regulation or exceeds the permitted use, you will need to obtain permission directly from the copyright holder. To view a copy of this license, visit http://creativecommons.org/licenses/by/4.0/. 
limit the therapeutic benefit ${ }^{9,10}$ or even introduce potentially pathogenic translocations ${ }^{11}$.

One recently developed technology that can overcome these problems is single base editing. Single base editors utilize a deaminase domain fused to a Cas9-nickase that can be directed by a single-guide RNA (sgRNA) to introduce targeted $C>T^{12}$ or $A>G^{13}$ conversions within a small, user-defined window. By enabling targeted mutagenesis without nuclease activity, base editors offer a promising platform for minimally disruptive and permanent exon-skipping therapies. We and others have previously demonstrated the application of $\mathrm{C}>\mathrm{T}$ base editors to induce programmable exon skipping through mutation of the conserved guanine residue preceding each exon, by targeting the cytosine on the opposite strand ${ }^{14,15}$. We estimated that the number of inner exons that can be targeted by this approach, termed CRISPR-SKIP, is 118,089 out of 187,636 inner exons. However, given the highly conserved sequence of splice acceptors, some CRISPR-SKIP target sites have low predicted on-target or high off-target scores ${ }^{14}$. In this work, we sought to increase the number of exons that can be targeted with high efficiency and specificity by adding a novel editing tool to the CRISPR-SKIP toolbox. More specifically, the experiments in this paper describe the application of $\mathrm{A}>$ $\mathrm{G}$ base editors (ABE) to induce exon skipping by mutating the adenine in the highly conserved AG dinucleotide within splice acceptors. Since therapeutic applications of exon skipping require achieving modification rates that surpass certain thresholds, we optimized the ABE performance by modifying the linker tethering the deaminase domain and the Cas9 scaffold. Finally, to enable in vivo applications of CRISPR-SKIP, we developed a split base editor architecture that is compatible with adenoassociated virus (AAV)-mediated delivery.

\section{Results}

Nearly all splice acceptors consist of a highly conserved adenosine-guanosine dinucleotide at the $5^{\prime}$ end of the exon (Fig. 1a) ${ }^{16}$. We hypothesized that conversion of the adenosine to a guanosine by targeting with $\mathrm{ABEs}^{13}$ would prevent recognition of the exon by the spliceosome, thereby triggering its exclusion from mature transcripts (Fig. 1b).

To test this hypothesis we targeted an ABE to the splice acceptor site of CTNNA1 exon 7. Plasmids encoding ABE 7.10, which consists of two engineered Escherichia coli TadA adenine deaminase domains fused to a Cas9-D10A nickase $^{13}$ and a sgRNA targeting the splice acceptor, were transfected into HEK293T cells. After 6 days, the RNA was isolated and retrotranscribed to cDNA, which was used in PCRs to detect skipping of exon 7. In samples treated simultaneously with the ABE and the sgRNA, two PCR amplicons were observed, corresponding to the expected size of the full-length mature mRNA and mRNA lacking exon 7. The transcript lacking exon 7 was not observed in samples treated with the sgRNA alone or the sgRNA in combination with dead Cas9 or Cas9-D10A (Fig. 2a). Sanger sequencing of the shorter PCR product confirmed that CTNNA1 exon 6 was followed immediately by exon 8 , confirming that exon 7 was skipped (Fig. 2b). High-throughput sequencing (HTS) of genomic DNA samples transfected with $A B E$ and the sgRNA confirmed successful $\mathrm{A}>\mathrm{G}$ mutation of the CTNNA1 exon 7 splice acceptor site in $6.52 \%$ of the strands (Fig. 2c).

To determine the optimal time frame needed to achieve maximal rates of exon skipping, we performed a timecourse experiment by transfecting HEK293T cells with plasmids encoding ABE 7.10 and the CTNNA1 exon 7 sgRNA and isolating RNA for analysis at various time points over a 10-day period. The truncated product was
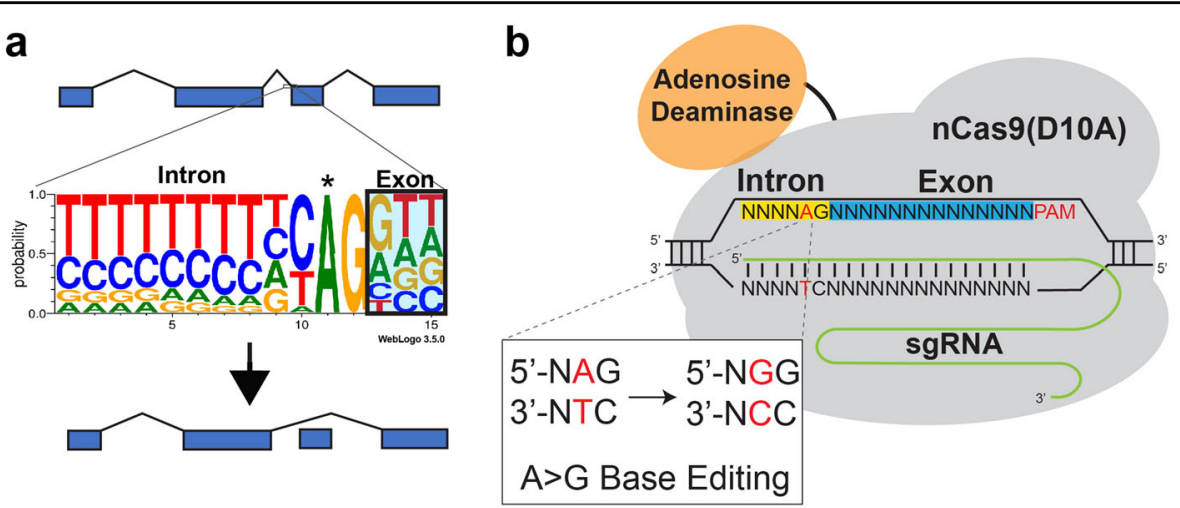

Fig. 1 Mutation of the conserved adenine residue of a splice acceptor using an ABE results in exon skipping. a Diagram of the consensus sequence of splice acceptor sites. Mutation of the conserved adenosine residue $\left(^{*}\right)$ prevents recognition by the splice machinery which leads to skipping of the exon. $\mathbf{b}$ ABEs can be directed to the splice acceptor site to convert the target $A$ to a $G$, which results in exon skipping 
a

\begin{tabular}{lcr|} 
Intron 7 & Exon 7 & PAM \\
CTGCAGAAACAAATCATTGTGG
\end{tabular}

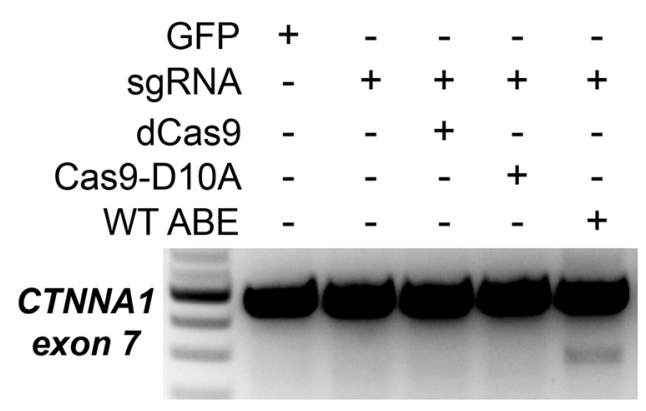

b

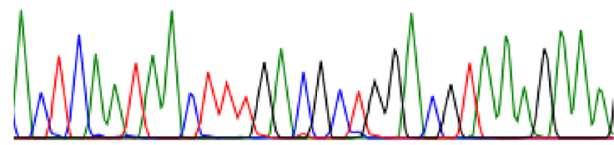

ACTCAATAACTTTGACGCTGGACGTAAAGAAA CTNNA1 Exon 6

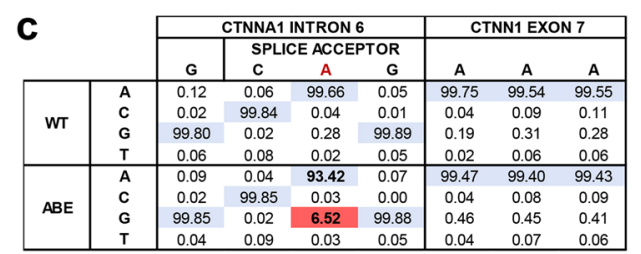

Fig. 2 Targeted exon skipping of CTNNA1 Exon 7 using ABE. a HEK293T cells were transfected with ABE 7.10 and a sgRNA targeting the splice acceptor site of CTNNA1 exon 7. Targeted exon skipping was observed after performing RT-PCR that could not be induced by the sgRNA alone, or in combination with dead Cas9 or D10A nickase Cas9. b Sanger sequencing of the shorter transcript confirmed exclusion of exon 7 from the CTNNA1 mature transcript. c High-throughput sequencing confirmed targeted $A>G$ mutations in genomic DNA at the CTNNA1 exon 7 splice acceptor site

readily detectable at day 2 and the rate of exon skipping continued to steadily increase until reaching a plateau at day 6 (Supplementary Fig. S1), which is similar to the optimal time frame for exon skipping with $\mathrm{C}>\mathrm{T}$ base editors ${ }^{14}$. For all subsequent experiments, samples were analyzed 6 days post transfection. In addition, the relative ratios of plasmids encoding the base editor and the sgRNA were varied to determine the optimal transfection conditions. We found that using $500 \mathrm{ng}$ of sgRNA plasmid in combination with $500 \mathrm{ng}$ of base editor plasmid or $250 \mathrm{ng}$ of base editor plasmid in combination with $750 \mathrm{ng}$ of sgRNA plasmid resulted in the highest rates of exon skipping in HEK293T cells transfected in 24-well plates (Supplementary Fig. S2).

To determine whether the exon skipping induced by $\mathrm{ABE}$ is cell line specific, we transfected HEPG2 and HCT116 cell lines with ABE, the CTNNA1 exon 7 sgRNA or an sgRNA targeting $A H C Y$ exon 9, respectively. In addition, to determine if the technique worked in other species, we transfected mouse Neuro2A and Hepa1-6 cells with $\mathrm{ABE}$ and a sgRNA targeting CTNNB1 exon 11. The target exon was skipped in all cell lines and only in the ABE-treated samples (Supplementary Fig. S3).

Since AON-induced exon skipping has already been successfully applied for correction of monogenic diseases such as Leber Congenital Amaurosis ${ }^{17}$, atherosclerosis ${ }^{18}$, FTDP- $17^{19}$, cancer $^{20}$, rheumatoid arthritis ${ }^{21}$, Huntington's disease ${ }^{5}$, dystrophic epidermis bullosa ${ }^{22}$, and Duchenne muscular dystrophy (DMD) ${ }^{23}$, we anticipate that CRISPR-SKIP will have multiple applications in biomedicine. However, correction of monogenic diseases requires production of a critical amount of functional protein product to achieve therapeutic benefit. Even though as little as $4 \%$ recovery of dystrophin expression restores significant muscle function to treat $\mathrm{DMD}^{24,25}$, a higher modification rate is needed in other cases, such as Huntington disease, which requires a $40 \%$ reduction of mutant Huntingtin for clinical improvement. For this reason, we sought to improve the exon-skipping rate induced by $\mathrm{ABE}$ by optimizing the amino-acid sequence of the linker between the TadA deaminase domains and the Cas9-D10A. Linkers between different domains in chimeric proteins influence parameters that are critical for protein function such as maintaining protein stability and folding ${ }^{26}$. To explore the effect of the linker domains on editing efficiency, we created ABE constructs with linkers of either five repeats of alanine followed by proline $\left(\mathrm{ABE}-\mathrm{AP}_{5}\right)$, five repeats of four glycine residues and a serine $\left(\mathrm{ABE}-\mathrm{GGGGS}_{5}\right.$ ), the linker in $\mathrm{ABE} 7 \cdot 10^{13}$ fused to GGGGS (ABE-Dual), or 5 repeats of glutamic acid followed by three alanine residues (ABE-EAAA 5 ) (Fig. 3a). Furthermore, since hypoxanthine is a spontaneous deamination product of adenine and a recently identified family of uracil-DNA glycosylases has been shown to act on hypoxanthine as part of the DNA repair process ${ }^{27}$, we reasoned that fusing $\mathrm{ABE}$ with a 83-amino acid uracil glycosylase inhibitor (UGI) domain may enhance DNA editing rate. For this reason, another construct was generated by adding a UGI domain to the C-terminus of the ABE 7.10 (ABE-UGI) (Fig. 3a).

These constructs were transfected separately into HEK293T cells along with the CTNNA1 exon 7 sgRNA, and rates of exon skipping were measured using RNA-seq (Fig. 3b). Our results demonstrated that $A B E$ with a GGGGS $_{5}$ linker $(7.73 \%, P=0.002)$ and the $\mathrm{EAAA}_{5}$ linker $(4.73 \%, P=0.082)$ induced exon skipping more efficiently than ABE 7.10 (3.73\%). Furthermore, ABE-UGI also outperformed the $A B E 7.10$ with a skipping efficiency of $7.73 \%(P=0.002)$.

In order to compare the editing efficiencies of these improved $\mathrm{ABE}$ variants across multiple targets, as well as 
a

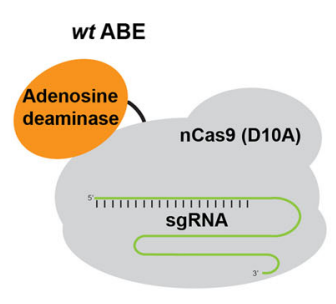

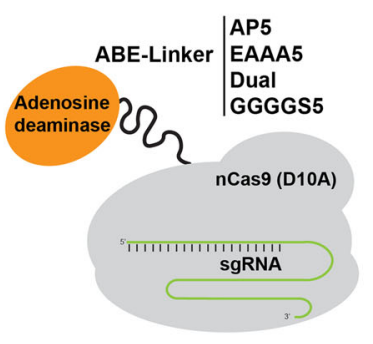
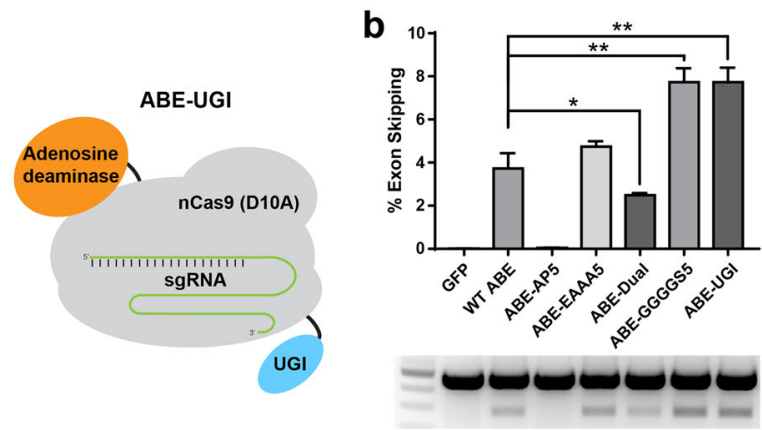

Fig. 3 Improvement of ABE editing activity by optimization of the linker between the deaminase and Cas9 or addition of a uracil glycosylase inhibitor domain. a Schematic representation of several of the ABE variants that were constructed by either modifying the linker tethering $\mathrm{nCas} 9$ and the deaminase domain or by fusing ABE 7.10 with a UGI. b High-throughput sequencing of cDNA demonstrated significantly increased levels of exon skipping by several of the ABE variants as compared to ABE 7.10. * and ** correspond to $P<0.05$ and $P<0.01$, respectively by two-tailed unpaired Student's $t$ test, $n=3$
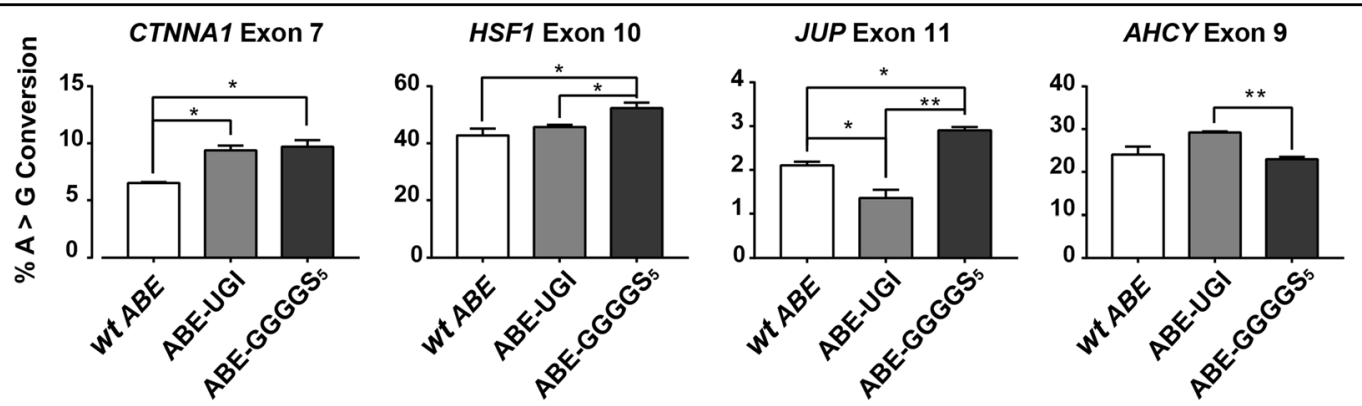

Fig. 4 Quantification of genomic DNA mutation rates created by several ABE constructs at multiple target sites. High-throughput sequencing was used to quantify rates of $A>G$ genomic DNA mutation and rates of exon skipping across multiple targets using several ABE variants. * and ** correspond to $P<0.05$ and $P<0.01$, respectively by two-tailed unpaired Student's $t$ test across two biological replicates
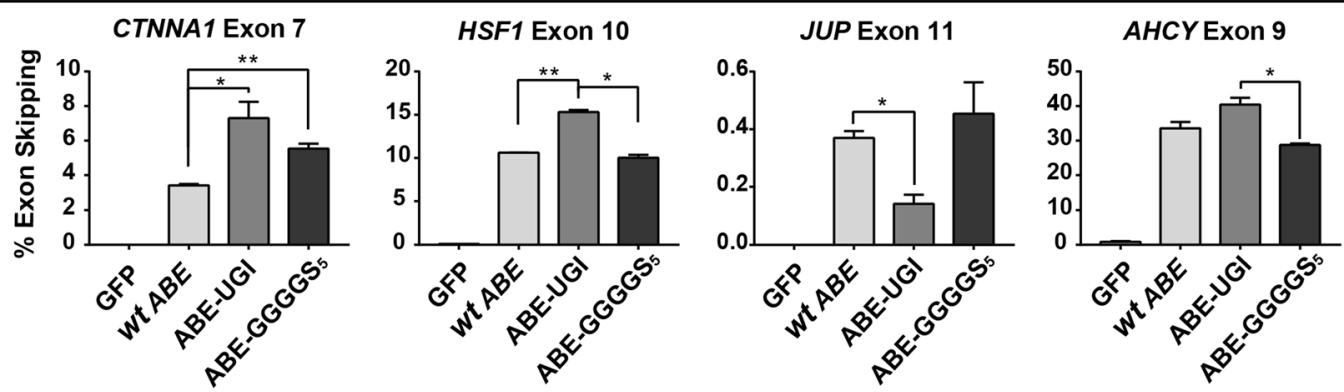

Fig. 5 Quantification of exon skipping rates at multiple gene targets induced by several ABE constructs. High-throughput sequencing of CDNA was used to quantify rates of exon skipping across multiple targets using several ABE variants. ${ }^{*}$ and ${ }^{* *}$ correspond to $P<0.05$ and $P<0.01$, respectively by two-tailed unpaired Student's $t$ test across two biological replicates

to correlate rates of modification in genomic DNA with rates of exclusion of the targeted exon from mRNA transcripts, we performed HTS on both genomic DNA (Fig. 4) and cDNA (Fig. 5) at multiple target sites in cells transfected with ABE 7.10, ABE-GGGGS , and ABE-UGI (Supplementary Table S1). These results confirmed that use of the ABE-GGGGS ${ }_{5}$ and ABE-UGI led to significant increases in both $A>G$ base editing rates and exonskipping rates over $A B E 7.10$ for many of the targets that were tested. In these experiments, the highest observed A $>\mathrm{G}$ mutation rates for each target were $9.70 \%$ by $\mathrm{ABE}$ GGGGS $_{5}$ at CTNNA1 exon 7, $52.33 \%$ by ABE-GGGGS ${ }_{5}$ at HSF1 exon 11, $2.90 \%$ by ABE-GGGGS ${ }_{5}$ at JUP exon 10 , and $29.23 \%$ by ABE-UGI at $A H C Y$ exon 9 (Fig. 4). The 
a
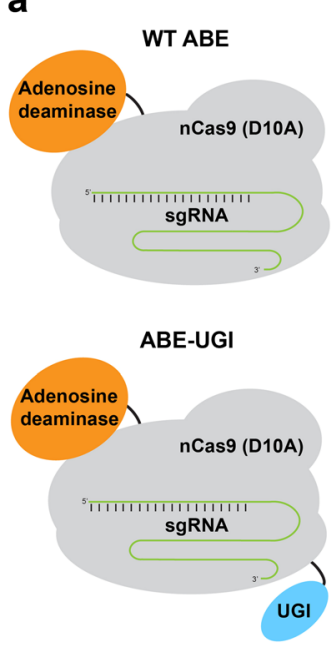
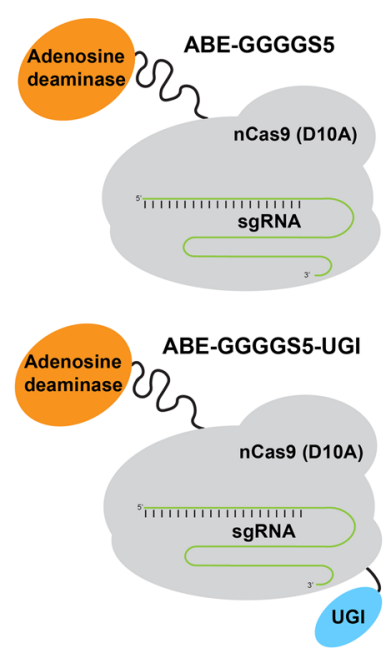

b
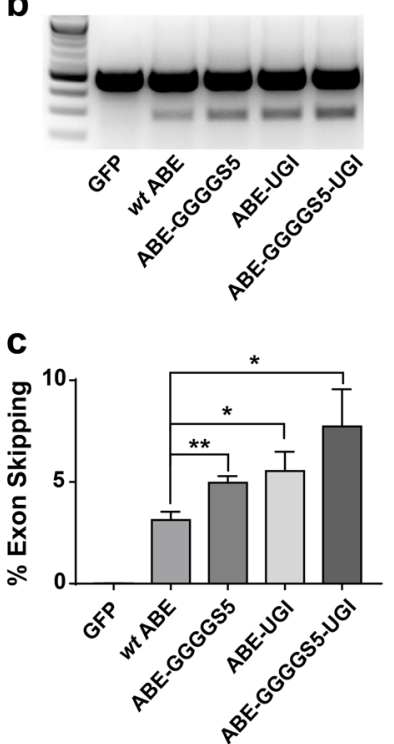

Fig. 6 Addition of both a uracil glycosylase inhibitor domain and an optimized linker further increased rates of exon skipping. a Schematic representation of the ABE variants constructed by either modifying the linker tethering $n C a s 9$ and the deaminase domain, by fusing ABE 7.10 with a UGl or both. $\mathbf{b}$ Combining the GGGGS 5 linker and UGI domain within the same ABE construct led to higher rates of exon skipping than the ABEs containing each modification individually, suggesting an increased $A>G$ mutation rates in genomic DNA when both domains are used. $\mathbf{c}$ Highthroughput sequencing analysis of RT-PCR products demonstrated significantly increased levels of exon skipping by several of the ABE variants compared with ABE 7.10. ( ${ }^{*}$ and ${ }^{* *}$ correspond to $P<0.05$ and $P<0.01$ respectively by two-tailed unpaired Student's $t$ test, $n=3$ )

highest observed exon-skipping rates as determined by RNA-seq for each target were $7.30 \%$ by ABE-UGI at CTNNA1 exon $7,15.31 \%$ by ABE-UGI at HSF1 exon 11, $0.45 \%$ by $\mathrm{ABE}-\mathrm{GGGGS}_{5}$ at JUP exon 10 and $40.45 \%$ by ABE-UGI at $A H C Y$ exon 9 (Fig. 5).

In an effort to further increase the editing efficiency of the $\mathrm{ABE}$, we created an additional $\mathrm{ABE}$ construct containing both the $\mathrm{GGGGS}_{5}$ linker and the UGI domain (ABEGGGGS $_{5}$-UGI) (Fig. 6a). We hypothesized that a base editor with both modifications would be more effective than either ABE-GGGGS ${ }_{5}$ or ABE-UGI. Plasmids encoding each ABE were transfected separately into HEK293T cells along with the CTNNA1 exon 7 sgRNA. Rates of exon skipping were measured by reverse transcription polymerase chain reaction (RT-PCR) (Fig. 6b) and compared using HTS (Fig. 6c). In this set of experiments, ABE-GGGGS $5_{5}$ UGI induced a higher rate of exon skipping than all other constructs tested with $7.73 \%$ compared to $3.13 \%$ for ABE 7.10 $(P=0.013), 4.96 \%$ for ABE-GGGGS $\quad(P=0.061)$, and $5.53 \%$ for ABE-UGI $(P=0.139)$.

We next sought to determine if the length of the linker between the deaminase domain and Cas9-D10A had any effect on the base editing window within the protospacer. We created ABE constructs with 1-7 repeats of the amino-acid sequence GGGGS. These constructs were then transfected into HEK293T cells along with one of two A-rich sgRNAs targeting the GAPDH locus
(Supplementary Table S4). After 3 days, genomic DNA was harvested and the editing rates of each of the As within the protospacer were evaluated for each construct (Supplementary Fig. S4). Interestingly, the editing window expanded towards the $5^{\prime}$ direction of the protospacer for each of the GGGGS constructs compared to ABE 7.10 and resulted in editing of the adenine in position 4, which was not observed with ABE 7.10. Furthermore, the editing efficiencies for positions 4 and 5 increased as the linker length decreased, with $\mathrm{ABE}-\mathrm{GGGGS}_{1}$ yielding the highest rates of base editing for these positions.

While improving editing efficiency addresses some of the limitations for therapeutic applications using singlebase editors, the large size of the DNA constructs encoding $A B E$ remains a significant roadblock to in vivo therapies that rely on gene delivery using AAVs. AAV offers a promising and safe delivery vehicle for gene therapy due to their ability to infect a broad range of cells, including nondividing cells, without eliciting more than a mild-immune response $\mathrm{e}^{28}$. In addition, they do not integrate into the host genome, thus reducing the risk of disrupting native gene function ${ }^{29}$. However, a major drawback of using AAVs is that the size of the transgene is limited to $4.7 \mathrm{~kb}$ for efficient expression ${ }^{30}$, which prevents the packaging of an $\mathrm{ABE}$.

One strategy that can be used to overcome these limitations is splitting the ABE transgene into two separate 


\section{a}

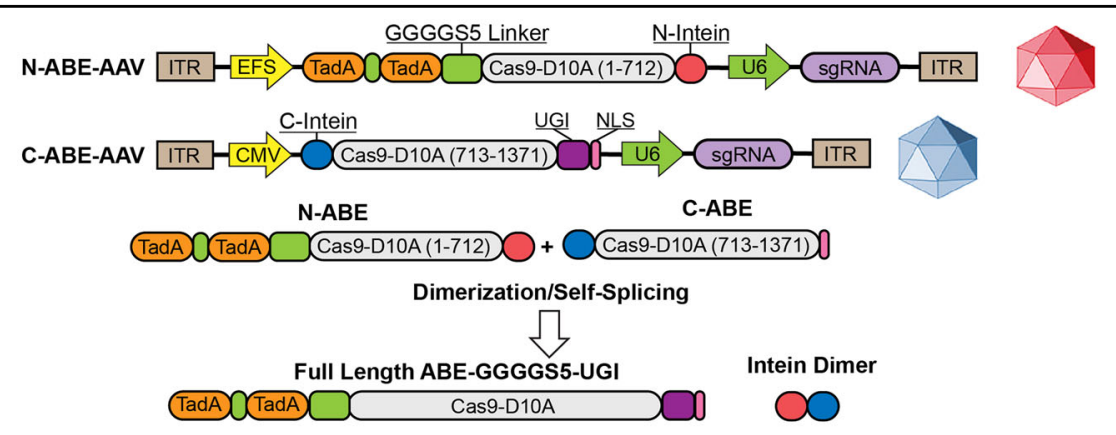

b
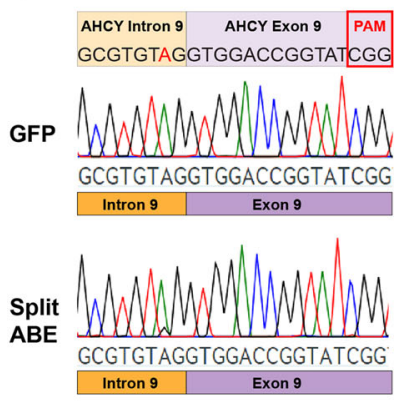

C

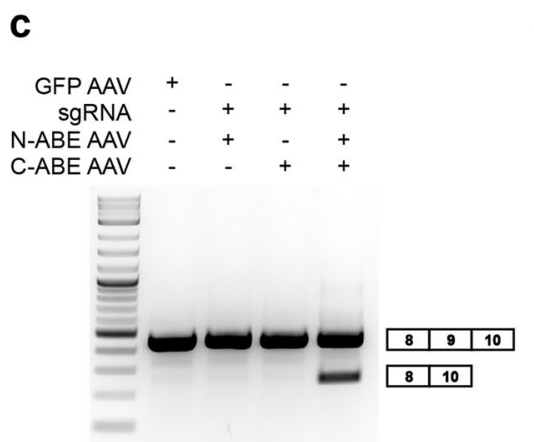

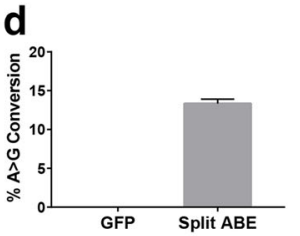

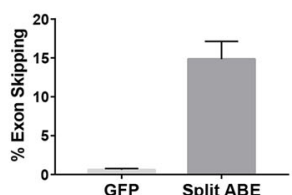

Fig. 7 Intein-mediated split ABE facilitates targeted exon skipping following delivery with AAV. a Schematics of the split-ABE AAV system. Nterminal and C-terminal intein sequences reconstitute the full-length protein when co-expressed within the cell. b Sanger sequencing traces from genomic DNA prepared from HEK293T cells transduced with either GFP-AAV or both N-ABE AAV and C-ABE AAV. A > G mutations are only observed when both N-ABE AAV and C-ABE AAV particles are delivered. c RT-PCR products confirm that exon skipping only occurs when both N-ABE AAV and C-ABE AAV are co-delivered. d Quantification of A > G mutation rates in the samples described in 7b using EditR $(n=3)$ and e exon-skipping rates by densitometry analysis of RT-PCR products $(n=3)$

vectors through the use of Rhodothermus marinus inteins, which when expressed as proteins are able to dimerize and cleave themselves out, leaving a near seamless fusion of the two gene products ${ }^{31}$. Here, we tested whether ABEs split in two separate expression cassettes using inteins are active in cultured cells. First, the ABE 7.10 open reading frame was split at the aspartic acid residue at amino-acid position 1109 into two plasmids. The N-terminal plasmid contained the TadA domains, the ABE 7.10 linker, and the first 712 amino acids of Cas9-D10A, followed immediately by an $\mathrm{N}$-terminal intein sequence (N-ABE). The second construct contained a $\mathrm{C}$-terminal intein sequence followed by the remaining 666 amino acids of ABE 7.10 (CABE) (Supplementary Fig. S5 and Supplemental Sequences). After transfecting HEK293T cells with the HSF1 exon 11 sgRNA with the N-ABE plasmid and C-ABE plasmid, we observed exon skipping only in the samples containing both $\mathrm{N}$ - and $\mathrm{C}$-terminus split base editor plasmids or the full-length $\mathrm{ABE}$ plasmid, which was transfected as control. We did not observe exon skipping levels above background in cells transfected with just the $\mathrm{N}$-terminus or the C-terminus split ABE (Supplementary Fig. S6). Surprisingly, RNA-seq revealed that the rate of exon skipping induced by split $\mathrm{ABE}(31.98 \%)$ was higher that the skipping rate measured in samples transfected with ABE 7.10 (26.23\%) $(P=0.0019)$, despite a potentially unfavorable reaction kinetic (Supplementary Fig. S7).

We then tested whether these constructs can be packaged into separate AAV particles and co-delivered to achieve base editing and subsequent exon skipping. We split the open reading frame of $\mathrm{ABE}-\mathrm{GGGGS}_{5}$-UGI at the same residue in Cas9-D10A as before, and cloned the separate constructs between AAV inverted terminal repeats (ITRs) (Fig. 7a). An sgRNA expression cassette under the control of a U6 promoter was also cloned between the ITRs of each construct to enable simultaneous delivery of the sgRNA. After cloning a sgRNA targeting AHCY exon 9 into these plasmids, they were packaged into AAV and used to transduce HEK293T cells. Cells were transduced with the $\mathrm{N}-\mathrm{ABE}$ AAV, the C-ABE AAV, or both. After 6 days we harvested the cells and confirmed $A>G$ mutations in genomic DNA and exon skipping only in the samples that were treated with both the N-ABE AAV and the C-ABE AAV (Fig. 7b, c). Analysis of genomic DNA from three independent experiments revealed $\mathrm{A}>\mathrm{G}$ modification rates of $13.33 \%$ (Fig. $7 \mathrm{~d}$ ), while densitometry analysis of RT-PCR products of the same samples revealed exon skipping rates of $14.85 \%$ (Fig. $7 \mathrm{e}$ ). 

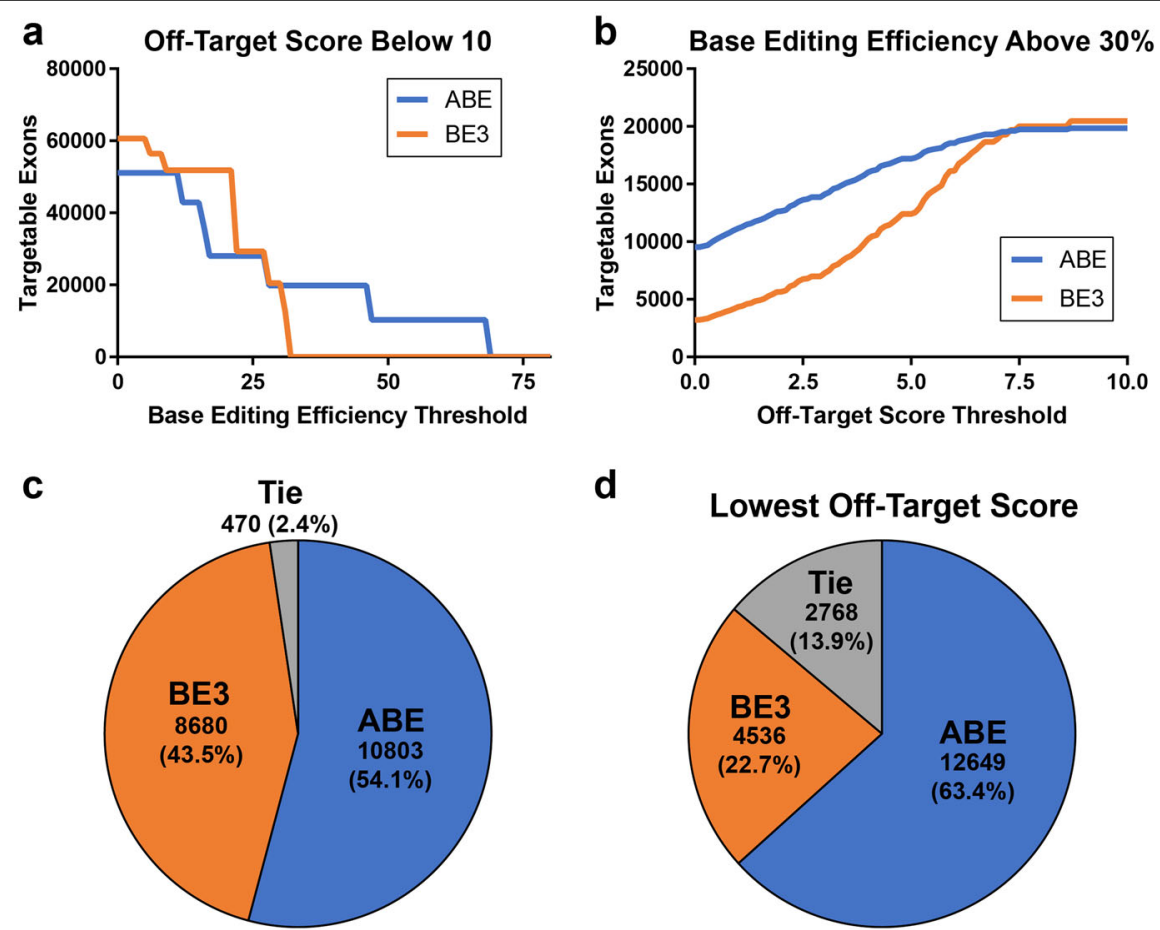

d

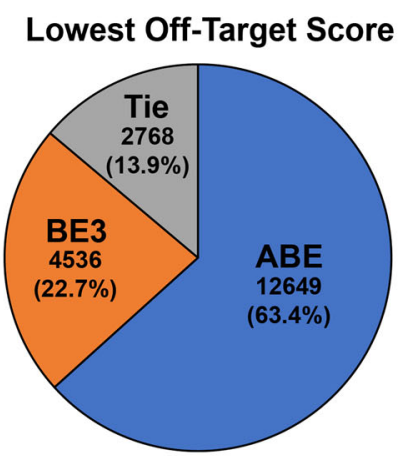

Highest Base Editing Efficiency

Fig. 8 ABE is predicted to have improved on-target and off-target editing efficiencies compared to BE3 when targeting splice acceptor sites. a Genome-wide computational analysis of the number of inner exons that can be targeted by ABE and BE3 with predicted editing efficiency of the target base at or above the value on the $x$-axis. Only sgRNAs with an off-target score below ten were considered. $\mathbf{b}$ Estimation of the number of inner exons that can be targeted by ABE and BE3 using sgRNAs with off-target scores at or below the value on the $x$-axis. Only sgRNAs with an ontarget base editing efficiency above 30\% were considered. c A total of 19,953 inner exons in the human genome can be targeted by both ABE and BE3. The sgRNAs needed to induce skipping with ABE have higher predicted base editing efficiency for targeting $54.1 \%$ of the exons, $\mathbf{d}$ and lower predicted off-target score for targeting $63.4 \%$ of the exons

To determine the contribution of $\mathrm{ABE}$ editors to the CRISPR-SKIP toolbox, we measured the number of inner exons that could be targeted by $\mathrm{ABE}$ using genome-wide computational analysis of PAMs compatible with exon skipping through mutation of the adenosine in the splice acceptor. In this analysis, when only highly specific sgRNAs with off-target scores ${ }^{32}$ at or below 10 were considered, we determined that the number of exons targetable by $\mathrm{ABE}$ is higher than the number of exons targeted by BE3 for all base editing efficiency thresholds over 30 (Fig. 8a). Furthermore, the numbers of exons that can be targeted by ABE with an off-target threshold lower than 7.5 is larger than the number that can be targeted with BE3 for on-target base editing efficiency above 30\% (Fig. 8b). There are 19,953 inner exons in the human genome that can be targeted by both $\mathrm{ABE}$ and $\mathrm{BE} 3$. $\mathrm{ABE}$ provides higher predicted efficiency for targeting 10,803 of these exons (54.1\%) (Fig. 8c) and higher specificity in 12,649 inner exons (63.4\%) (Fig. 8d). These results support that ABE not only expands the number of exons that can be targeted by CRISPR-SKIP, but also enables increasing the efficiency and specificity of CRISPR-SKIP.

Collectively, the work described in this paper establishes a platform for inducing exon skipping by mutation of the conserved adenosine in splice acceptors. Since this modification is introduced in genomic DNA, the resulting alternative splicing is expected to be permanent, which provides an important therapeutic advantage over exonskipping oligonucleotides. However, one of the concerns with gene editing technologies, including base editing, has been the possibility of unintended off-target mutations in regions of the genome that are similar to the target sequence $^{33}$. To investigate the incidence of off-target mutations we analyzed genomic DNA at four predicted off-target locations ${ }^{32}$ for each sgRNA tested by HTS to detect possible mutations (Supplementary Table S2). We only observed off-target $A>G$ mutations at one site within a noncoding region, which was introduced by the $J U P$ exon 10 sgRNA. Notably, this sgRNA had the highest predicted off-target score of all sgRNA tested in this work and the mutation rate was low $(\sim 0.5 \%)$. 


\section{Discussion}

The work described in this paper significantly improves the CRISPR-SKIP toolbox not only by increasing the number of potential exons that can be targeted, but also by engineering a base editing system that is compatible with in vivo delivery by AAV.

Mutation of the adenosine in the conserved splice acceptor AG dinucleotide preceding the exon was predicted to prevent recognition of the targeted exon by the spliceosome machinery; therefore, we anticipated a linear relationship between splice acceptor site mutation rate and exon-skipping rate across targets. However, this was not the case. For example, at $A H C Y$ exon 9 mutation rates of $\sim 20 \%$ in genomic DNA resulted in skipping rates of $\sim 50 \%$, whereas at HSF1 exon 11, mutation rates of $\sim 50 \%$ in genomic DNA resulted in rates of exon skipping of only $\sim 20 \%$. Since one of the major blocks during transcript elongation is the splicing junction ${ }^{34-36}$, which leads to transient polymerase pausing at the splice sites ${ }^{37}$, it is reasonable to expect that the rate of exon skipping can be higher than the conversion rate measured in genomic DNA. While this is what we observed at most targets, the opposite was observed when we targeted HSF1. One potential explanation is that $H S F 1$ is expressed at low levels in HEK293T cells and it is possible that a time frame longer than 6 days might be needed for the changes in genomic DNA to be reflected in the transcriptome. While it is difficult to predict the exact reason for the overall lack of correlation, we noted that when we studied each target site individually, there was indeed a correlation between the rate of mutation in genomic DNA and exon skipping in mRNA, with higher rates of splice acceptor mutations corresponding to higher rates of exon skipping for all targets except for HSF1 (Supplementary Fig. S8).

Overall, the observed genomic DNA modification rates and exon skipping rates appeared to vary widely among targets. By analyzing the on-target activity of each guide using $w t$ active Cas9, we observed that each of the guides was able to create indels at rates ranging from $\sim 11 \%$ to $\sim 30 \%$ at the target site demonstrating that each sgRNA was active (Supplementary Table S3). However, wt Cas9 nuclease activity did not correlate with $\mathrm{ABE}$ editing rates when using the same sgRNA, thus suggesting that the adenine deaminase domain imposes other editing restrictions that are not well understood yet while underscoring the need to perform further studies to develop computational tools for more effectively predicting optimal $A B E$ target sites. While the results of our in silico screening in Fig. 8 show that $A>G$ base editors often have greater predicted on-target editing efficiency and better predicted off-target editing profiles than BE3, it is important to evaluate both base editors at the target site experimentally, as actual results will likely be dependent on local sequence and chromatin context.
Interestingly, the linker optimization studies that we performed identified $\mathrm{ABE}$ variants that were more active than the ABE 7.10. However, the improvement was target specific, and we did not identify one single linker variant that was more effective across all targets. It is worth noting that the shorter GGGGS linker variants are able to achieve higher rates of exon skipping for bases located toward the $5^{\prime}$ of the protospacer compared to the longer GGGGS linker variants or ABE 7.10. These results suggest that the best variant to use is dependent on the position of the target A within the protospacer and the sequence context, and could explain why the greatest increases in base editing and exon skipping were seen for CTNNA1 exon 7 and JUP exon 11, as the splice acceptor adenine was located at position 5 of the protospacer, while the target adenine for HSF1 exon 10 and $A H C Y$ exon 9 was located at positions 6 and 7, respectively.

Importantly, we also demonstrated that coupling UGI at the $\mathrm{C}$-terminus of $\mathrm{ABE}$ increases editing efficiency, similar to what has been observed using $\mathrm{C}>\mathrm{T}$ base editors. While this observation was not confirmed at all target sites, one possible explanation could be that the UGI prevents removal of the hypoxanthine created by the adenine deaminase. Should this explanation prove true, it emphasizes the importance of manipulating the DNA repair pathways to achieve improved editing rates. These findings also suggest that further improvements can potentially be accomplished by fusing ABE to other protein domains that regulate DNA repair.

Other groups have recently accomplished improved modification rates using $\mathrm{C}>\mathrm{T}$ base editors by implementing codon optimization algorithms that removed potential polyadenylation sequences from the Cas9 open reading frame as well as adding additional nuclear localization signals ${ }^{38}$. The ABE that we used in these experiments has been codon optimized and we did not identify any polyadenylation signals within Cas9; however, inclusion of additional nuclear localization signals could improve further the editing efficiency, which might be particularly important for therapeutic applications requiring high levels of correction.

Adenine base editors were used recently in vivo by AAV delivery to correct a mutation that causes DMD in the $m d x$ mouse model ${ }^{39}$. However, this work was performed using a dual trans-splicing AAV system, which relies on ITR homology and whose efficiency is typically considered limited. While we have not performed a direct comparison between this approach and the intein-based split system that we developed, it is noteworthy that our split base editor architecture appears to be at least as efficient as ABE 7.10 in vitro. This result was unexpected because the intein system requires assembly of the full-length $A B E$ and excision of the intein after translation and before editing can occur and, therefore, the kinetics of the 
reaction are less favorable than that of $\mathrm{ABE}$ 7.10. However, it is possible that expression of two shorter transcripts from two independent promoters increases the efficiency of transcription and translation of $\mathrm{ABE}$, thus offsetting a potentially slower editing reaction. It remains to be determined whether this system is also efficient in vivo after systemic AAV administration, although the data from Ryu et al. ${ }^{39}$ supports that reconstitution in vivo after delivery from two different viral particles is possible.

In summary, the ability to disrupt splice acceptor sites using adenine base editors further expands the available tools for inducing therapeutic exon skipping. It proves especially useful for exon targets that are not accessible by BE3 due to PAM restrictions and further increases the total amount of exons that can be skipped using single-base editors. In addition, when both $\mathrm{ABE}$ and $\mathrm{BE} 3$ can be used to target the same exon, $\mathrm{ABE}$ generally offers higher on-target activity and lower off-target activity. These improved ABE variants and the split $\mathrm{ABE}$ architecture that we developed represent significant progress towards enabling in vivo base editing studies since the potential to package a base editor into AAV particles is an advancement that will enable exploration of various therapies, which will have a lasting impact on the field of gene therapy.

\section{Materials and methods}

\section{Plasmids and cloning}

The plasmid used for SpCas9 sgRNA expression and expression of SpCas9, dCas9, and Cas9-D10A were gifts from Charles Gersbach. The ABE 7.10 plasmid was generated through Gibson assembly of a gBlock Gene Fragment (Integrated DNA Technologies) containing the TadA domains and ABE 7.10 linker, as described by Gaudelli et al. ${ }^{13}$, into a Cas9-D10A backbone (Addgene plasmid \#41816). The ABE plasmids containing the various linkers described in Fig. 2A were created through Gibson assembly of gBlock Gene Fragments into the ABE 7.10 plasmid (Supplemental Sequences). The ABE-UGI plasmid was generated through Gibson assembly of the TadA deaminase domains into an spCas9-BE3 plasmid (pCMV-BE3) that was a gift from David Liu (Addgene plasmid \#73021). Split-ABE constructs were generated through Gibson assembly of gBlock Gene Fragments. Amino acid sequences are provided in Supplemental Sequences. All base editor constructs were under the control of the CMV promoter, except for N-ABE-AAV which was under the control of an EFS promoter ${ }^{40}$.

All oligonucleotides used in this work were obtained from IDT. The oligonucleotides for sgRNA generation were hybridized, phosphorylated and cloned into the SpCas9 sgRNA vector using BbsI sites ${ }^{41,42}$. Guide sequences are provided in Supplementary Table S4. All sgRNA cassettes were under control of the human U6 promoter.

\section{Cell culture and transfection}

HEK293T, HCT116, Neuro2A, and Hepa1-6 cell lines were obtained from the American Tissue Collection Center (ATCC). They were maintained in DMEM supplemented with $10 \%$ fetal bovine serum and $1 \%$ penicillin/ streptomycin at $37^{\circ} \mathrm{C}$ with $5 \% \mathrm{CO}_{2}$. Transfections were performed in 24-well plates using Lipofectamine 2000 (Invitrogen) following manufacturer's instructions. The amount of DNA used for lipofection was $1 \mu \mathrm{g}$ per well. Transfection efficiency was routinely higher than $80 \%$ as determined by fluorescent microscopy following delivery of a control GFP-expression plasmid.

\section{AAV vector production}

HEK293T cells were seeded in $15 \mathrm{~cm}$ dishes and transfected at $80-90 \%$ confluence. GFP-AAV plasmid, N$\mathrm{ABE}-\mathrm{AAV}$ or C-ABE-AAV were transfected along with pHelper and pAAV-DJ from the AAV-DJ Packaging System from Cell Biolabs in a 1:1:1 ratio using calcium phosphate and a total of $60 \mu \mathrm{g}$ per plate. Media was replaced $24 \mathrm{~h}$ post transfection. Cell pellets were harvested at $72 \mathrm{~h}$ post transfection through manual cell scraping and centrifuged at $1500 \times g$ for $12 \mathrm{~min}$. After aspirating the supernatant, the cell pellet was resuspended in $1 \mathrm{~mL}$ AAV lysis buffer $(50 \mathrm{mM}$ Tris- $\mathrm{HCl} \mathrm{pH}=8.5$, $150 \mathrm{mM} \mathrm{NaCl}$ and $2 \mathrm{mM} \mathrm{MgCl}_{2}$ ). Resuspended pellets were subjected to three freeze-thaw cycles between an ethanol/dry ice bath and a $37^{\circ} \mathrm{C}$ water bath. Lysed cell pellets were then spun at $10,000 \times g$ for $10 \mathrm{~min}$ and the supernatant was collected as crude lysate. Lysates were then treated with $50 \mathrm{U}$ benzonase per $\mathrm{mL}$ and incubated at $37^{\circ} \mathrm{C}$ for $30 \mathrm{~min}$ to digest unpackaged plasmid. Crude lysates were added directly to cells or flash frozen with liquid nitrogen and stored at $-80{ }^{\circ} \mathrm{C}$ for future use.

\section{AAV infection}

HEK293T cells were infected in suspension in the wells of a 24-well plate by mixing $100 \mu \mathrm{L}$ of crude lysate with 20,000 cells in $150 \mu \mathrm{L}$ of cell culture medium. In the case of the samples containing both N-ABE AAV and C-ABE AAV, $50 \mu \mathrm{L}$ of each lysate was added. Protamine sulfate was added to the lysate-cell mix at a final concentration of $5 \mu \mathrm{g} / \mathrm{mL}$ to enhance infection efficiency. Cells were incubated for $24 \mathrm{~h}$ at which point the media was aspirated and replaced with $500 \mu \mathrm{L}$ of fresh medium. Infected cells were incubated for a total of 6 days before harvesting genomic DNA and RNA for analysis.

\section{RT-PCR}

RNA was harvested from cell pellets using the RNeasy Plus Mini Kit (Qiagen) according to manufacturer's instructions. cDNA synthesis was performed using the qScript cDNA Synthesis Kit (Quanta Biosciences) from 500-1000 ng of RNA with cycling conditions performed 
as directed by the supplier. PCR was performed using KAPA2G Robust PCR kits from Kapa Biosystems. The $25 \mu \mathrm{L}$ reactions used $50 \mathrm{ng}$ of cDNA, Buffer A $(5 \mu \mathrm{L})$, Enhancer $(5 \mu \mathrm{L})$, dNTPs $(0.5 \mu \mathrm{L}), 10 \mu \mathrm{M}$ forward primer $(1.25 \mu \mathrm{L}), 10 \mu \mathrm{M}$ reverse primer $(1.25 \mu \mathrm{L})$, KAPA2G Robust DNA Polymerase (0.5 U), and water (up to $25 \mu \mathrm{L}$ ). We used cycling parameters as recommended by the manufacturer. The PCR products were visualized in $2 \%$ agarose gels stained with ethidium bromide and images were captured using a ChemiDoc-It2 (UVP). The DNA sequences of the primers for each target are provided in Supplementary Table S5.

\section{Densitometry analysis}

Skipping efficiencies were determined by densitometry analysis of the PCR products obtained from RT-PCR and analyzed by agarose gel electrophoresis using Image J software. After subtracting background noise, band intensity was compared using the following formula: $\%$ Exon skipping $=\frac{\text { Skipped band intensity }}{\text { wt Band intensity }+ \text { Skipped band intensity }}$, where band intensity is the sum of each pixel grayscale value within the selected area of the band.

\section{Amplification of genomic DNA}

Genomic DNA was isolated using a DNeasy Blood and Tissue Kit (Qiagen) and PCR amplification was performed with KAPA2G Robust PCR kits (KAPA Biosystems) as described above, using 20-100 ng of template DNA.

\section{Editing window analysis using Sanger sequencing and EditR software}

Genomic DNA from samples treated with an ABEGGGGS variant and an A-rich sgRNA was amplified using the PCR primers listed in Supplementary Table S5. Sanger sequencing of the PCR amplicons was performed by the W.M. Keck Center for Comparative and Functional Genomics at the University of Illinois at UrbanaChampaign using the primers listed in Supplementary Table S6. Base editing efficiencies were estimated by analyzing the sanger sequencing traces using $\operatorname{Edit}^{43}$.

\section{High-throughput sequencing}

HTS was performed on PCR amplicons from genomic DNA or RNA harvested from duplicate transfections of HEK293T cells. PCR primers for HTS are provided in Supplementary Table S7. After validating the quality of PCR products by gel electrophoresis the PCR products were isolated by gel extraction using the Zymoclean Gel DNA Recovery Kit (Zymo Research). Indexed HTS amplicon libraries for samples described in Figs. 4 and 5 were prepared with the Hyper Library construction kit from Kapa Biosystems without shearing. Indexed HTS amplicon libraries for samples described in Figs. 6 and 7 were prepared using a Nextera XT DNA Library Prep Kit
(Illumina). The libraries were quantitated by qPCR and sequenced on MiSeq Nano flowcells for 251 cycles from each end of the fragments using a MiSeq 500-cycle sequencing kit version 2. Fastq files were generated and demultiplexed with the bcl2fastq v2.17.1.14 Conversion Software (Illumina). All sequencing was performed by the W.M. Keck Center for Comparative and Functional Genomics at the University of Illinois at UrbanaChampaign. All sequencing data are available at Gene Expression Omnibus under Series GSE120125.

\section{Sequence analysis}

DNA and RNA sequencing reads were demultiplexed by PCR primer sequences and quality trimmed to Phred quality score 20 at the $3^{\prime}$ end using cutadapt. Read pairs with at least one mate trimmed to $50 \mathrm{bp}$ or less were discarded. DNA reads were then aligned to the human genome version GRCh38 using Bowtie $2^{44}$. To determine on-target and off-target base editing rates, alternative allele depths were calculated by Samtools mpileup over $120 \mathrm{bp}$ windows centered around the protospacer sequences for on and off targets. A global estimate of sequencing error was made by averaging the fraction of alternative allele depths across all positions. A positiondependent estimate of sequencing error was determined by fraction of alternative allele depth at each genomic position. Significant $\mathrm{A}>\mathrm{G}$ or $\mathrm{T}>\mathrm{C}$ conversion was determined by using the one-sided binomial test at a $p$ value cutoff of $10^{-5}$, using the higher of the global or position-dependent sequencing error estimates as the background probability of nucleotide conversions. Indel rates were calculated using Mutect $2^{45}$.

Reads from paired-end RNA-seq were mapped to the human genome version GRCh38 with TopHat $^{46}$ for isoform quantification. Forward and reverse reads were combined as a single read for analysis. Reads displaying the exon-skipped junction were counted toward the exonskipped transcript and reads displaying either the $5^{\prime}$ or $3^{\prime}$ canonical splice junction were counted towards the canonical isoform. Reads that did not display any of the previously mentioned splice junctions were excluded from quantification. The exon-skipping rate for each biological duplicate was calculated by dividing the number of exonskipped transcript reads by the sum of the number of exon-skipped and canonical transcript reads. Estimates of the overall exon-skipping rates were made by averaging duplicates.

\section{Genome-wide targetability analysis}

All exons of protein coding transcripts (genomic assembly GRCh38, GENCODE release 26) that are not the first or last exon in a transcript were scanned for PAMs in the appropriate range. The predicted position-dependent base editing efficiencies for BE3 are identical to those used 
in Gapinske et al. ${ }^{14}$. The corresponding efficiency values for ABE were estimated from ABE7.8 efficiency values from Gaudelli et al. ${ }^{13}$, Fig. 3c and Supplemental Fig. S7b by the following method: first, the maximum base editing efficiency was estimated by taking the highest observed editing efficiency across all $\mathrm{ABE}$ variants and sites from Fig. 3c; then, the relative base editing efficiencies of ABE7.8 from Supplemental Fig. S7b positions 4-9 were multiplied by the estimated maximum base editing efficiency to obtain the estimated position-dependent base editing efficiencies for ABE.

For each candidate sgRNA, the entire genome was scanned for all sequences with at most two mismatches and an off-target score was calculated ${ }^{34}$. Any sgRNA with an off-target score above ten was removed.

\section{Acknowledgements}

This work was supported by the ZJU-Illinois Institute Research Program, an American Heart Association Scientist Development Grant (17SDG33650087), $\mathrm{NIH}$ R03EB026064 and NIH R01GM127497 to P.P. and NIH R01CA163336 and NSF 0822613 to J.S.S. This material is based upon work supported by the National Science Foundation Graduate Research Fellowship Program under Grant no. DGE-1746047 to M.G.

\section{Author details}

'Department of Bioengineering, University of Illinois at Urbana-Champaign, Urbana, IL 61801, USA. ${ }^{2}$ Department of Physics, University of Illinois at UrbanaChampaign, Urbana, IL 61801, USA. ${ }^{3}$ Carl R. Woese Institute for Genomic Biology, University of Illinois at Urbana-Champaign, Urbana, IL 61801, USA. ${ }^{4}$ Carle Illinois College of Medicine, Champaign, IL 61820, USA. ${ }^{5}$ Cancer Center at Illinois, University of Illinois at Urbana-Champaign, Urbana, IL 61801, USA

\section{Author contributions}

J.W., A.L., M.G., W.W., J.S.S. and P.P. designed the experiments. J.W., M.G., S.M. S.S. and W.W. performed the experiments. J.W., A.L., M.G., S.M., S.S., W.W., J.S.S. and P.P. analyzed the data and J.W., A.L., J.S.S. and P.P. wrote the paper.

\section{Conflict of interest}

J.W., M.G., W.W. and P.P. are named inventors on patent applications related to gene editing and exon skipping with base editors. The remaining authors declare that they have no conflict of interest.

\section{Publisher's note}

Springer Nature remains neutral with regard to jurisdictional claims in published maps and institutional affiliations.

Supplementary Information accompanies the paper at (https://doi.org/ 10.1038/541421-019-0109-7).

Received: 24 September 2018 Revised: 28 June 2019 Accepted: 2 July 2019 Published online: 20 August 2019

\section{References}

1. Pan, Q., Shai, O., Lee, L. J., Frey, B. J. \& Blencowe, B. J. Deep surveying of alternative splicing complexity in the human transcriptome by highthroughput sequencing. Nat. Genet. 40, 1413-1415 (2008).

2. Kole, R. \& Krieg, A. M. Exon skipping therapy for Duchenne muscular dystrophy. Adv. Drug Deliv. Rev. 87, 104-107 (2015).

3. Kowalewski, C. et al. Amelioration of junctional epidermolysis bullosa due to exon skipping. Br. J. Dermatol. 174, 1375-1379 (2016).

4. Burghes, A. H. \& McGovern, V. L. Antisense oligonucleotides and spinal muscular atrophy: skipping along. Genes Dev. 24, 1574-1579 (2010).
5. Crooke, S. T. Molecular mechanisms of action of antisense drugs. Biochim. Biophys. Acta 1489, 31-44 (1999).

6. Mou, $\mathrm{H}$. et al. CRISPR/Cas9-mediated genome editing induces exon skipping by alternative splicing or exon deletion. Genome Biol. 18, 108 (2017).

7. Long, C. et al. Correction of diverse muscular dystrophy mutations in human engineered heart muscle by single-site genome editing. Sci. Adv. 4, eaap9004 (2018).

8. Ceccaldi, R., Rondinelli, B. \& D'Andrea, A. D. Repair pathway choices and consequences at the double-strand break. Trends Cell Biol. 26, 52-64 (2016).

9. Haapaniemi, E., Botla, S., Persson, J., Schmierer, B. \& Taipale, J. CRISPR-Cas9 genome editing induces a p53-mediated DNA damage response. Nat. Med. 24, 927-930 (2018)

10. Ihry, R. J. et al. p53 inhibits CRISPR-Cas9 engineering in human pluripotent stem cells. Nat. Med. 24, 939-946 (2018).

11. Kosicki, M., Tomberg, K. \& Bradley, A. Repair of double-strand breaks induced by CRISPR-Cas9 leads to large deletions and complex rearrangements. Nat. Biotechnol. 36, 765-771 (2018).

12. Komor, A. C., Kim, Y. B., Packer, M. S., Zuris, J. A. \& Liu, D. R. Programmable editing of a target base in genomic DNA without double-stranded DNA cleavage. Nature 533, 420-424 (2016).

13. Gaudelli, N. M. et al. Programmable base editing of $A^{*} T$ to $G^{*} C$ in genomic DNA without DNA cleavage. Nature 551, 464-471 (2017).

14. Gapinske, M. et al. CRISPR-SKIP: programmable gene splicing with single base editors. Genome Biol. 19, 107 (2018).

15. Yuan, J. et al. Genetic modulation of rna splicing with a crispr-guided cytidine deaminase. Mol. Cell 72, 380-394 (2018).

16. Sibley, C. R., Blazquez, L. \& Ule, J. Lessons from non-canonical splicing. Nat. Rev. Genet. 17, 407-421 (2016)

17. Gerard, X. et al. AON-mediated exon skipping restores ciliation in fibroblasts harboring the common leber congenital amaurosis cep290 mutation. Mol. Ther. Nucleic Acids 1, e29 (2012).

18. Khoo, B., Roca, X., Chew, S. L. \& Krainer, A. R. Antisense oligonucleotide-induced alternative splicing of the APOB mRNA generates a novel isoform of APOB. BMC Mol. Biol. 8, 3 (2007).

19. Kalbfuss, B., Mabon, S. A. \& Misteli, T. Correction of alternative splicing of Tau in frontotemporal dementia and Parkinsonism linked to chromosome 17. J. Biol. Chem. 276, 42986-42993 (2001).

20. Mercatante, D. R., Mohler, J. L. \& Kole, R. Cellular response to an antisensemediated shift of Bcl-x pre-mRNA splicing and antineoplastic agents. J. Biol. Chem. 277, 49374-49382 (2002)

21. Karras, J. G., McKay, R. A., Dean, N. M. \& Monia, B. P. Deletion of individual exons and induction of soluble murine interleukin-5 receptor-alpha chain expression through antisense oligonucleotide-mediated redirection of pre-mRNA splicing. Mol. Pharmacol. 58, 380-387 (2000).

22. Goto, M. et al. Targeted skipping of a single exon harboring a premature termination codon mutation: implications and potential for gene correction therapy for selective dystrophic epidermolysis bullosa patients. J. Invest. Dermatol. 126, 2614-2620 (2006).

23. Aartsma-Rus, A. \& van Ommen, G. J. B. Antisense-mediated exon skipping: a versatile tool with therapeutic and research applications. RNA 13, 1609-1624 (2007).

24. van Putten, M. et al. Low dystrophin levels increase survival and improve muscle pathology and function in dystrophin/utrophin double-knockout mice. FASEB J. 27, 2484-2495 (2013).

25. Dawson, T. M., Li, D., Yue, Y. \& Duan, D. Marginal level dystrophin expression improves clinical outcome in a strain of dystrophin/utrophin double knockout mice. PLoS ONE 5, e15286 (2010).

26. Chen, X., Zaro, J. L. \& Shen, W. C. Fusion protein linkers: property, design and functionality. Adv. Drug Deliv. Rev. 65, 1357-1369 (2013).

27. Lee, H. W., Dominy, B. N. \& Cao, W. New family of deamination repair enzymes in uracil-DNA glycosylase superfamily. J. Biol. Chem. 286, 31282-31287 (2011).

28. Daya, S. \& Berns, K. I. Gene therapy using adeno-associated virus vectors. Clin. Microbiol. Rev. 21, 583-593 (2008).

29. Penaud-Budloo, M. et al. Adeno-associated virus vector genomes persist as episomal chromatin in primate muscle. J. Virol. 82, 7875-7885 (2008).

30. Wu, Z., Yang, H. \& Colosi, P. Effect of genome size on AAV vector packaging. Mol. Ther. 18, 80-86 (2010).

31. Chew, W. L. et al. A multifunctional AAV-CRISPR-Cas9 and its host response Nat. Methods 13, 868-874 (2016).

32. Hsu, P. D. et al. DNA targeting specificity of RNA-guided Cas9 nucleases. Nat. Biotechnol. 31, 827-832 (2013). 
33. Fu, Y. et al. High-frequency off-target mutagenesis induced by CRISPR-Cas nucleases in human cells. Nat. Biotechnol. 31, 822-826 (2013).

34. Kwak, H., Fuda, N. J., Core, L. J. \& Lis, J. T. Precise maps of RNA polymerase reveal how promoters direct initiation and pausing. Science 339, 950-953 (2013).

35. Fuchs, G. et al. 4sUDRB-seq: measuring genomewide transcriptional elongation rates and initiation frequencies within cells. Genome Biol. 15, R69 (2014).

36. Veloso, A. et al. Rate of elongation by RNA polymerase II is associated with specific gene features and epigenetic modifications. Genome Res. 24, 896-905 (2014).

37. Alexander, R. D., Innocente, S. A., Barrass, J. D. \& Beggs, J. D. Splicing-dependent RNA polymerase pausing in yeast. Mol. Cell 40, 582-593 (2010).

38. Zafra, M. P. et al. Optimized baseeditors enable efficient editing in cells, organoids and mice. Nat. Biotechnol. 36, 888-893 (2018).

39. Ryu, S. M. et al. Adenine base editing in mouse embryos and an adult mouse model of Duchenne muscular dystrophy. Nat. Biotechnol. 36, 536-539 (2018).
40. Tabebordbar, M. et al. In vivo gene editing in dystrophic mouse muscle and muscle stem cells. Science 351, 407-411 (2016).

41. Brown, A., Woods, W. S. \& Perez-Pinera, P. Multiplexed targeted genome engineering using a universal nuclease-assisted vector integration system. ACS Synth. Biol. 5, 582-588 (2016).

42. Gapinske, M., Tague, N., Winter, J., Underhill, G. H. \& Perez-Pinera, P. Targeted gene knock out using nuclease-assisted vector integration: hemi- and homozygous deletion of JAG1. Methods Mol. Biol. 1772, 233-248 (2018).

43. Kluesner, M. G. et al. EditR: a method to quantify base editing from Sanger sequencing. CRISPR J. 1, 239-250 (2018).

44. Langmead, B. \& Salzberg, S. L. Fast gapped-read alignment with Bowtie 2. Nat. Methods 9, 357-359 (2012).

45. Cibulskis, $\mathrm{K}$. et al. Sensitive detection of somatic point mutations in impure and heterogeneous cancer samples. Nat. Biotechnol. 31, 213-219 (2013).

46. Kim, D. et al. TopHat2: accurate alignment of transcriptomes in the presence of insertions, deletions and gene fusions. Genome Biol. 14, R36 (2013). 